present fluffy masses on the anterior surface of the collarette. Septic teeth were found and, after extraction of these, the irido-cyclitis cleared up completely under treatment.

Extraction of the now mature cataract was contemplated but on examination with the slit-lamp microscope, the lens was found to be tremulous. The iris was not tremulous so that there was no dislocation of the lens; the tremulousness of the lens, however, was of the same quality as that of a tremulous iris; on the slightest movement of the eyes, the lens trembled as if it were suspended on an elastic string.

\title{
Comment
}

I can only interpret this phenomenon as being due to fluidity of the vitreous; the attack of irido-cyclitis was a severe one and at its conclusion left the eye with a slightly softer intra-ocular tension than the other eye. I have not found any reference to tremulousness of the lens in such literature as I have been able to procure.

\section{TUBERCULOSIS OF THE CHOROID ASSOCIATED WITH GENERALIZED MILIARY TUBERCULOSIS*}

\author{
BY \\ FREDERICK TOOKE \\ MONTREAL, CANADA
}

For many years past the allegiance of oculists has been challenged. The question at issue has been, which has provided the greater source of scientific satisfaction, a foundation upon which a future superstructure of a complete ophthalmic expression can ultimately be raised, the Eye Hospital, Infirmary, or Institute, or the Ophthalmic Clinic as an integral unit in the various elements which form the regularly standardized or constituted hospital of to-day.

For the practising ophthalmologist, and much more for the self-styled ophthalmic surgeon, a great deal is to be said for the Infirmary in supplying in quantities operative material without which no surgeon can approach his subject with assurance, develop the delicacy and refinement of a surgical technique which the specialty imperatively demands, or in an entirely ethical sense by introducing him to the community at large through the medium

\footnotetext{
* Read before the American Ophthalmological Society, July, 1935.
} 
of his public service. The same statement holds true regarding the diagnosis and treatment of what we have been pleased to term external diseases; often the expression of some remote systemic disorder, unfortunately only too frequently regarded by the ophthalmologist in a surgical sense and treated as such.

But our ophthalmologist must to-day be much more than an ophthalmic surgeon. He must approach the solution of his sometimes extremely complex riddle by more devious channels of investigation and interrogation than is the case possibly in any other department of modern medicine. For such an individual who as a student demands a completely rounded out enquiry into a relatively small number of cases, his sources of information must be open and near at hand.

With such a preamble as an oculist attached to a department in a general hospital, may I presume to argue that a great deal of most valuable and interesting scientific information is very frequently provided for the seeker after truth, from the various elements of a hospital in which a Department of Ophthalmology exists. The consideration of the case which I am about to report would bear out such an argument. The almost daily observation of the patient by my associates in the service supporting my observations would strongly emphasize the stand I have presumed to take.

J. D., a male infant of thirteen months was admitted to the Paediatric Department of the Royal Victoria Hospital, Montreal, December 22, 1934, and died January 14, 1935, twenty-three days later. Three days prior to admission the child had appeared to be in his usual good health, but he suddenly became irritable and restless. He refused part of his breast feeding and his sleep was broken. There had been three attacks of vomiting, and the patient seemed to be feverish. During this period he had suffered from a series of convulsions which were invariably induced when he was disturbed or moved; one of these occurred at home lasting for three minutes when his physician was in attendance. The child became quite pale, the eyes and head were turned to the right, the body was limp, and the fingers were extended. During the convulsion and after it Chovestek's sign was present; between the convulsions the patient was relatively happy, comfortable and contented. He laughed, played, and did not appear to be sick.

From a personal and family history there did not appear to be any predisposition towards tuberculosis from the child's forebears, but an old friend, who was very suspiciously tuberculous had frequently been in attendance on the child when the mother required assistance.

On admission to the hospital another characteristic convulsion 
took place almost at once, after which the temperature registered $101^{\circ}$. The pupils were regular, and of equal size, and reacted to light; the fundi were normal. The anterior cervical glands were bilaterally enlarged, while the posterior cervicals were small and shotty. An examination of the chest showed it to be symmetrical with regular movements on respiration; the percussion note was resonant throughout, breath sounds were clear and no

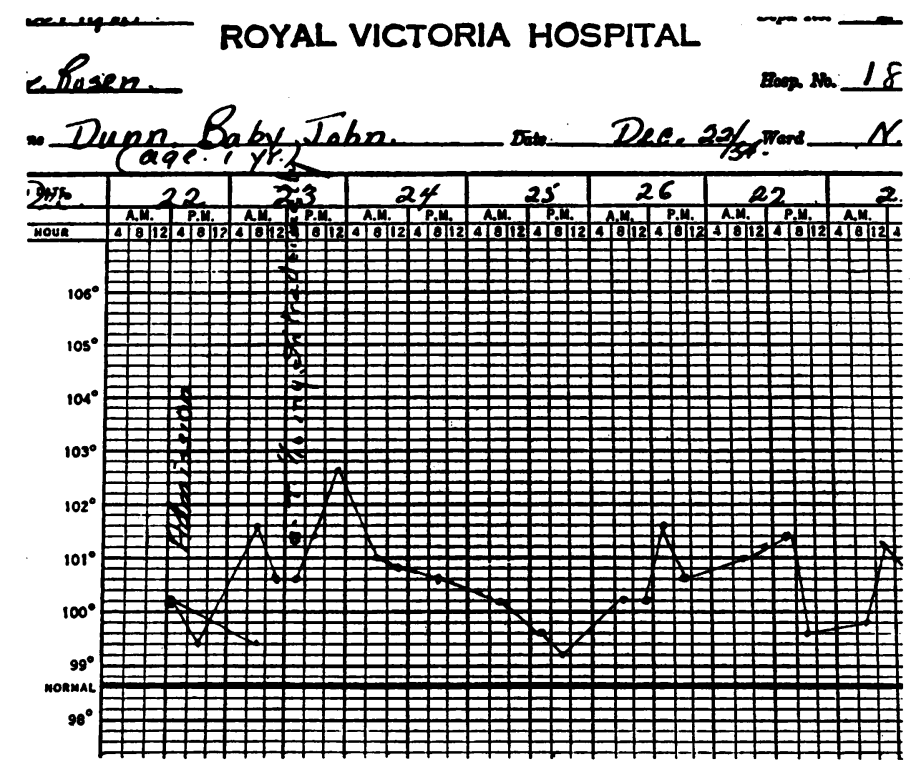

FiG. 1.

Typical tuberculin reaction following intradermal injection of $1 / 10 \mathrm{mg}$. O.T. Temperature after its initial fall gradually rose to $105^{\circ}$ at exitus.

râles were heard. The spleen, however, was enlarged and soft. The tendon reflexes were generally increased, while the abdominal reflexes were absent. The tonsils were enlarged.

A lumbar puncture was performed and an absolutely clear fluid was withdrawn containing only two cells per c.mm. The Pandy test was negative. There was no stiffness of the neck and Kernig's sign was absent. The day following admission $1 / 10 \mathrm{mgm}$. of O.T. was administered intra-dermally with a typical local and general reaction (Fig. 1), while an X-ray photograph of the chest showed the lungs to be peppered or mottled with innumerable miliary tubercles (Fig. 2). Four days following admission a lumbar puncture was again performed when eight c.c. of clear fluid were withdrawn; there were only six cells per c.mm., and pressure 
could not be recorded. Five days following admission sputum was recovered from stomach washings and tubercle bacilli were demonstrated.

When I was called upon to examine the fundi at this date I detected a crop of characteristic tubercles of the choroid in each eye. A maintained examination could not possibly be carried out owing to the child's age, on account of its precarious health, and also because the introduction of bright light created a retinal hyperaesthesia. There were at least seven tubercles

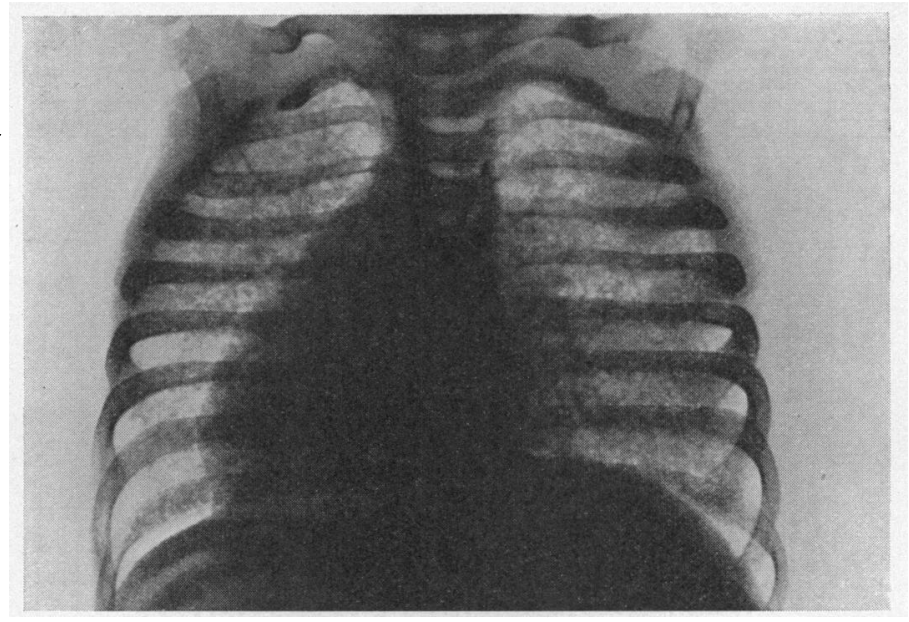

FIG. 2.

$\mathrm{X}$-ray photograph of chest showing generalized miliary tuberculosis of lungs.

in the right fundus and five in the left; probably more. They were situated at the temporal sides of the disc margins just before one arrives at the macular area ; there was one tubercle observed further to the temporal side and above and beyond the macular area in the right fundus oculi. The tubercles measured from one to two millimetres in diameter and could best be described as occurring as a cluster or a group. They did not select the tissue underlying the bifurcation of the retinal vessels as is so frequently the case; indeed the retinal vessels were absolutely uninvolved in the clinical picture. The disc surfaces were of good colour and their margins were sharply outlined, quite unlike the blurred margins of discs frequently associated with choroidal tubercles occurring after an advanced tuberculous meningitis has been established clinically.

The two lumbar punctures carried out up to date would appear to have excluded the meningeal factor. On January 8 , or six days prior to death, a lumbar puncture was again performed when three 
to four c.c. of clear fluid were withdrawn showing only ten cells per c.mm. Pandy negative. Four days prior to death there was no stiffness of the neck. 'The patient's eyes were repeatedly examined up to two days prior to exitus and the picture originally revealed in the fundi showed little if any variation from that which I have already described. Temperature at the time of death rose to $105^{\circ}$.

A general autopsy was performed and revealed a diffuse tuberculous involvement of all the parenchymatous organs. There was a caseous focus demonstrated in a right peribronchial lymph gland. Grossly meningeal involvement was at first questioned, but on examining the meninges along the Sylvian fissure, tubercles were noted; they would appear, however, to have been but few. What was of greatest significance was that on sectioning the brain there was revealed an area of caseation involving the cortex of the left parietal region ; a primary focus ante-dating the appearance of the tubercles on the meningeal capsule or sheath. Autopsy also

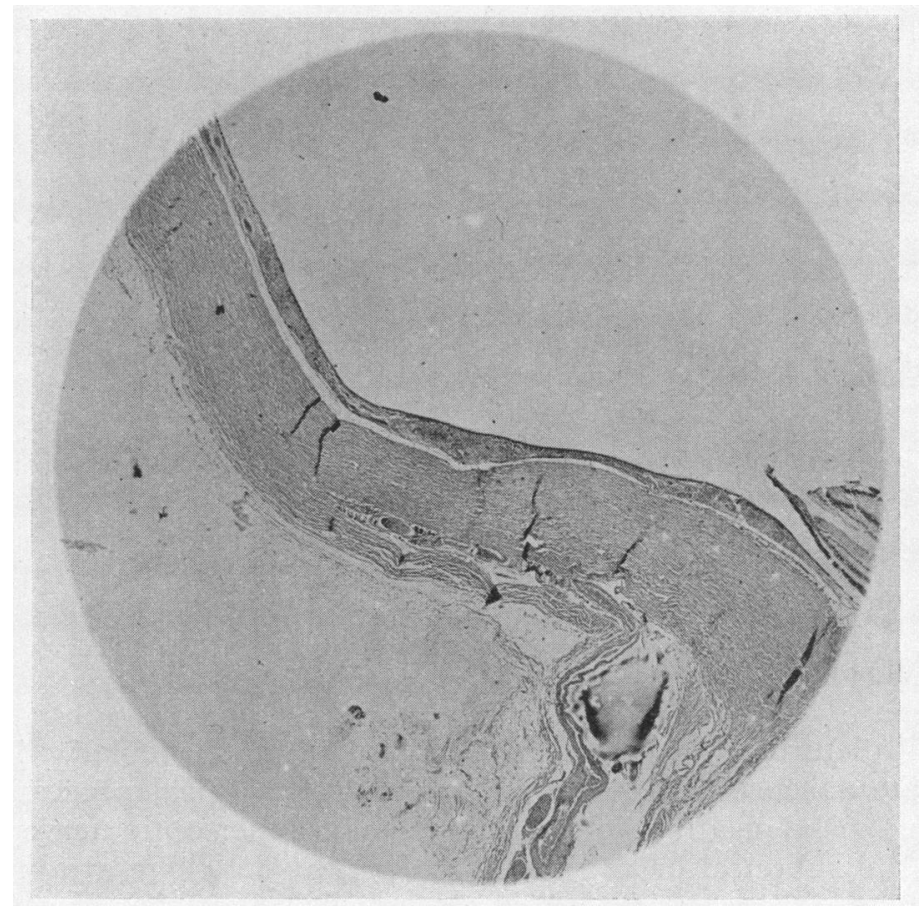

FIG. 3.

Low power magnification. Shows at least three tubercles or tuberculous foci. Absolute absence of involvement of vaginal space and optic nerve sheath. 
revealed extensive miliary tuberculosis involving the lungs, liver, spleen, kidneys, adrenals, thoracic and abdominal lymph glands, pancreas and myocardium, with exudative ulcertive tuberculous enteritis.

The posterior half of each eye was excised and sections were made in paraffin.

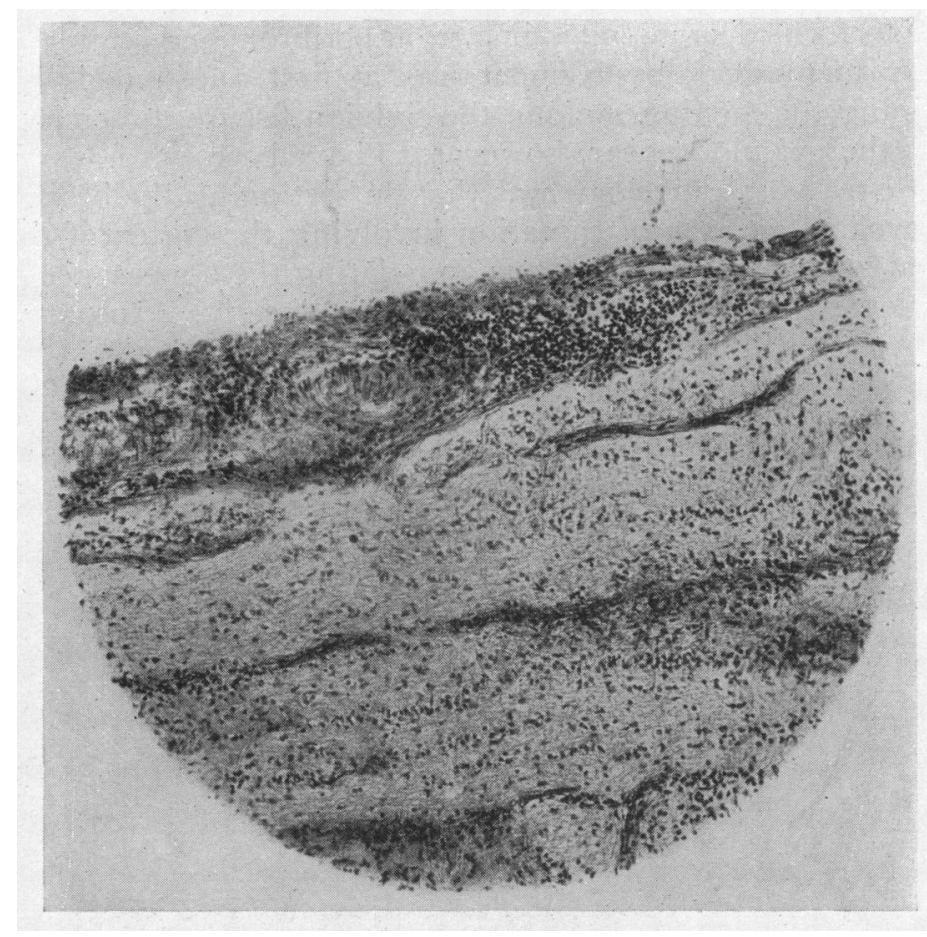

FIG. 4.

High power magnification. Longitudinal section of optic nerve showing infiltration of nerve fibres through contact with involved pia-arachnoid.

The picture which attracts one's attention particularly is the absolute absence of association of any pathological process purporting to be of a meningeal character in either optic nerve itself or in the subdural or vaginal space (Fig. 3). There are no free cells in this space and the pia-arachnoid is peculiarly free from an associated leucocytic infiltration. I am showing by way of contrast a section of a blurred optic nerve occurring with a choroidal tubercle which appeared in a case of advanced tuberculous meningitis shortly before death (Fig. 4). Here, microscopically, 
one can note the unquestionable infiltration of the outermost nerve fibres obviously associated with the pathological changes in the meninges or in the cerebro-spinal fluid contained within the vaginal space.

In the sections obtained and considered seriatim one can see what can better be described as four tuberculous areas rather than

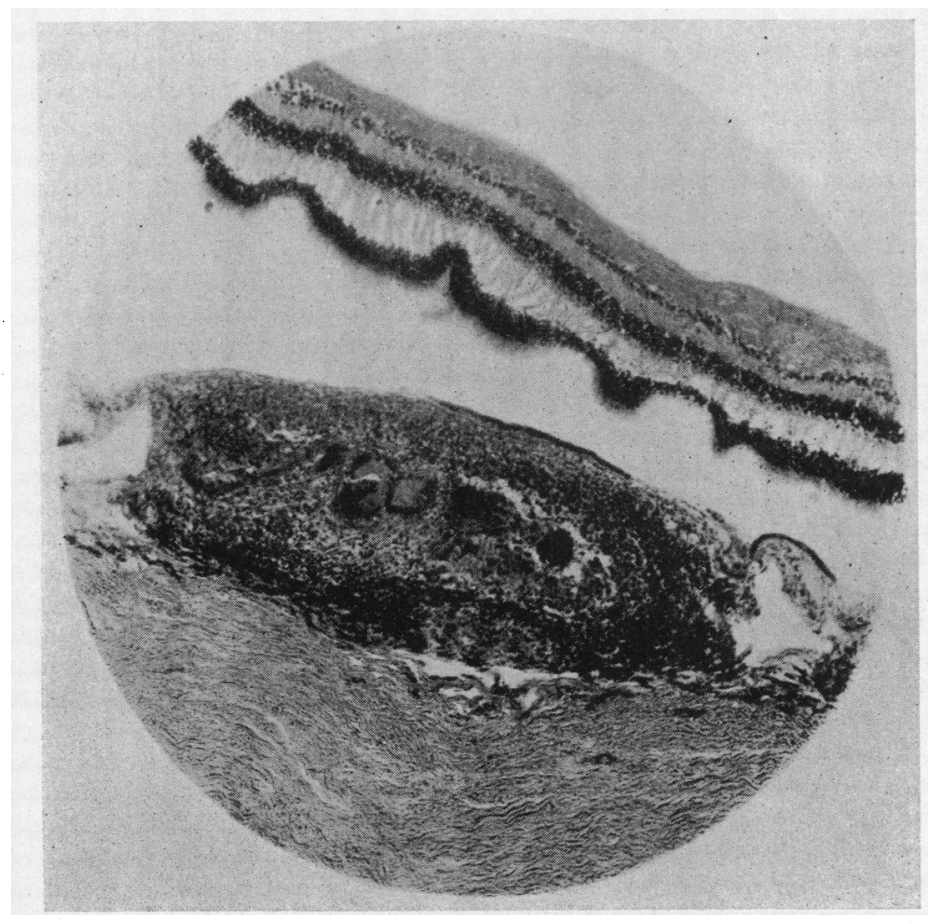

Fig. 5.

High power magnification. Recent tubercle with dense circumscribed infiltration of leucocytes encapsulating area of tuberculous tissue.

what one is accustomed to acknowledge or recognize as actual tubercle formations (Fig. 3). In a true tubercle with Bielschowsky's stain one would expect to find a trabecular formation of the tubercle proper formed by collagen fibres, the whole zone to be surrounded by a limiting capsule of lymphocytes and fibrinous exudate including or walling off the other well recognized component parts of the tubercle proper. Here such a picture is not afforded one, and there is no such thing as a sharply limited zone of peri-tubercular infiltration discernible (Fig. 5). In fact the 
acute phase of an intense perivasculitis with the establishment of perivascular tubercles about the capillaries is but very feebly in evidence. The choroidal vessels, it is true, are decidedly dilated but one cannot note any degree of perivasculitis. There are quantities of epithelioid cells, giant cells without number, and the condition of granulation tissue with coagulation necrosis which

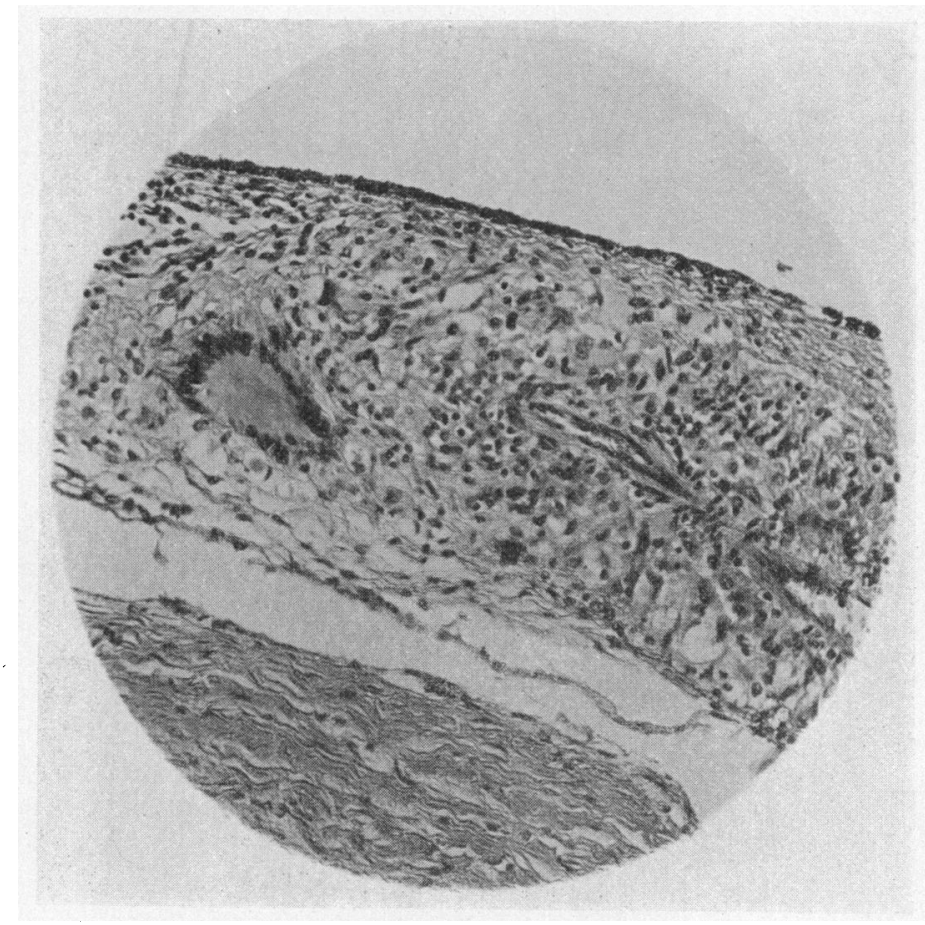

FIG. 6.

High, power magnification. Shows chronic tuberculous formation without encapsulation as in recent tubercle formation. Epithelioid cells, coagulation necrosis and giant cells well marked. Very little perivasculitis.

would indicate an established rather than an incipient lesion still in the process of extension, but relatively a chronic rather than an acute picture pathologically regarded. The whole process was limited within the membrane of Bruch (Fig. 6). Tubercle bacilli were demonstrated in the tissues.

In reviewing the clinical and pathological facts in so far as I have been able to establish and record them, the question arises how far are we in error in associating the establishment of 
tubercles of the choroid with a co-existing problematic, or for that matter even with an actually existing, tuberculous meningitis rather than with the underlying early or miliary type of a generalized tuberculous infection carried by the blood stream to various foci prior to an involvement of the meninges. The choroidal tubercle may be no more a complication or an extension of a meningeal tuberculosis than it is one of a tuberculosis involving the peritoneum, pleura, pericardium or any other membrane enclosing a serous cavity within which an active tuberculous process may be, clinically at least, evident and manifesting expression.

Rich and McCordock (J. Hopkins Bulletin, 1933) in their excellent article have reported upon the gross pathological findings of eighty-two cases of tuberculous meningitis not necessarily associated in so far as I have been able to learn with tubercles in the choroid such as those which I am reporting. A review of their conclusions has a very direct bearing on the case under consideration. In the vast majority of their cases, in seventy-seven out of eighty-two, older tuberculous foci were detected in the adjacent brain tissue, the bones of the cranial cavity, or about the spinal cord, or in the meninges themselves. In other words, the condition of meningitis was not brought about through the blood stream but rather through direct contact of an old caseous or necrotic area with the meninges or with the cerebro-spinal fluid adjacent to it. The same argument holds true regarding the cerebro-spinal fluid affecting the fibres of the optic nerve in the section that I have already shown (Fig. 4). It is but natural to enquire regarding the origin of the local areas of necrosis assumed to be responsible for the meningitis as in the present case the tuberculoma of the left cerebrum; undoubtedly it was the result of the early dissemination of bacilli which so frequently follows upon a primary infection. No matter what the site of a progressive lesion may have been, let us assume the peribronchial glands, a scattering of bacilli to distant organs through the blood stream has been generally demonstrated; such a dissemination resulting oft-times in sparsely scattered tuberculous masses, "solitary tubercles" of varying size with or without competent incapsulation which I demonstrated to have occurred in the choroid (Fig. 6). Many of these foci have become sterile; in others bacilli remain alive as I have demonstrated in my section; but in the presence of acquired resistance or relative chronicity their power of multiplication has been held in abeyance and the lesion has remained quiescent as was most certainly the case during the last ten days of this patient's life.

Early dissemination, which occurs before an acquired resistance has been established following a primary infection, results in areas 
of caseation which extend to infect the meninges in infancy and early childhood.

Miliary tuberculosis has so commonly been associated with tuberculous meningitis in the very young that the latter has long been assumed to be simply a manifestation of the former. One should stress not so much the coincidence of a meningitis with miliary tuberculosis as the coincidence of a meningitis with a low degree of acquired resistance. In brief, again to quote Rich and McCordock, the sequence of events is not (1) primary lesion, (2) meningitis occurring as a part of a miliary tuberculosis; but rather (1) primary lesion, (2) dissemination of bacilli leading to formation of caseous foci in structures adjacent to the meninges with or without frank miliary tuberculosis, (3) discharge of bacilli from these foci into the meninges causing meningitis. These authors have shown that isolated tubercles of the brain or meninges arising as part of a slowly progressive miliary tuberculosis may at times, as in this particular instance, resolve into caseous foci large enough to infect the cerebro-spinal fluid by discharging large numbers of bacilli directly into it. One must regard the development of a meningitis as a fortuitous event, depending not only upon the existence of a generalized miliary tuberculosis but upon the chance extension of infection from an established caseous focus which happened to develop adjacent to the meninges.

\section{Summary}

1. Tubercles of the choroid are established as a haematogenous infection in a condition of a generalized miliary tuberculosis.

2. Tuberculous meningitis results from contact with necrotic foci from adjacent tissues earlier involved through the blood stream.

3. Tuberculous meningitis is a late rather than an early manifestation of the course of the disease.

4. Tubercles of the choroid in certain cases are coincident rather than complicating manifestations of tuberculous meningitis.

5. Tubercles of the choroid may exist not because of, but in spite of, tuberculous meningitis. 